\title{
Simultaneous observation of viscoelastic deformation and induced magnetic anisotropy in [Co1x(FeNi)x]75Si15B10 metallic glasses
}

\author{
Fernández-Gubieda, M. L.; Barandiarán, J. M.; Nielsen, Otto V
}

Published in:

Journal of Applied Physics

Link to article, DOI:

$10.1063 / 1.339432$

Publication date:

1987

Document Version

Publisher's PDF, also known as Version of record

Link back to DTU Orbit

Citation (APA):

Fernández-Gubieda, M. L., Barandiarán, J. M., \& Nielsen, O. V. (1987). Simultaneous observation of viscoelastic deformation and induced magnetic anisotropy in [Co1x(FeNi)x]75Si15B10 metallic glasses. Journal of Applied Physics, 62(6), 2579-2582. https://doi.org/10.1063/1.339432

\section{General rights}

Copyright and moral rights for the publications made accessible in the public portal are retained by the authors and/or other copyright owners and it is a condition of accessing publications that users recognise and abide by the legal requirements associated with these rights.

- Users may download and print one copy of any publication from the public portal for the purpose of private study or research.

- You may not further distribute the material or use it for any profit-making activity or commercial gain

- You may freely distribute the URL identifying the publication in the public portal 
different and the creation or annihilation of dangling bonds is not the cause of the light-induced excess conductivity. Our results also do not contain an exact mechanism of light-induced excess conductivity, but they allow us to make some suggestions. Since the effect only occurs in compensated samples, we believe it does not involve either isolated donor or acceptor states. A possible interpretation is that the effect is caused by the states of boron-phosphorus complexes. By time-of-flight photoconductivity measurements Marshall, Street, and Thompson ${ }^{4}$ showed that the compensation introduces a distinct band of localized states near the band edges, and they suggested that these states are boron-phosphorus complexes. As electrons absorb photons and are subsequently trapped at the localized states of boron-phosphorus complexes, the dark conductivity and photoconductivity will increase after light exposure. Thus, the threshold photon energy $(<0.95 \mathrm{eV})$ needed to create the effect is much less than the energy gap $(1.7 \mathrm{eV})$. Upon annealing at $180^{\circ} \mathrm{C}$, the electrons trapped in the boron-phosphorus complexes are thermally emptied from the traps, and hence the conductivity goes back to state $\mathrm{A}$.

In summary, our photon energy dependence measurements confirm that the mechanism of light-induced effect for compensated samples is different from those for undoped samples. We believe the most likely explanation is the introduction of localized states originating from boron-phosphorus complexes.

The authors would like to thank Dr. K. N. Huang and T. J. Sano for their help in experiments. This work is partially supported by the National Science Council of the Republic of China.

'D. L. Staebler and C. R. Wronski, J. Appl. Phys. 51, 3262 (1980)

${ }^{2}$ H. Mell and W. Beyer, J. Non-Cryst. Solids 59, 405 (1983).

${ }^{3}$ J. F. Tian, D. S. Jiang, B. R. Zeng, Lin Huang, G. L. Kong, and L. Y. Lin, Solid State Commun. 57, 543 (1986).

${ }^{4} J$. M. Marshall, R. A. Street, and M. J. Thompson, Phys. Rev. B 29, 2331 (1984).

\title{
Simultaneous observation of viscoelastic deformation and induced magnetic anisotropy in $\left[\mathrm{Co}_{1-x}(\mathrm{FeNi})_{x}\right]_{75} \mathrm{Si}_{15} \mathrm{~B}_{10}$ metallic glasses
}

\author{
M. L. Fernández-Gubieda and J. M. Barandiarán \\ Facultad de Ciencias, Universidad del Pais Vasco Apartado 644, 48080 Bilbao, Spain \\ O. V. Nielsen \\ Department of Electrophysics, The Technical University of Denmark, DK-2800 Lyngby, Denmark
}

(Received 5 January 1987; accepted for publication 13 May 1987)

\begin{abstract}
Amorphous ribbons of composition $\left[\mathrm{Co}_{1-x}(\mathrm{FeNi})_{x}\right]_{75} \mathrm{Si}_{15} \mathrm{~B}_{10}(0.1<x<0.7)$ have been annealed by means of short current pulses while applying a tensile stress. Current densities in the range 3-5 $10^{7} \mathrm{~A} \mathrm{~m}^{-2}$ have been used. Viscoelastic deformation and induced magnetic anisotropy were determined simultaneously after the treatments at room temperature. The induced anisotropy consists of two contributions, one irreversible and the other recoverable. The strength of the anisotropy and the deformation depends on the anneal temperature, the thermal history of the sample and the composition. The composition dependence of the plastic deformation and the induced anisotropy is similar and the influence of preannealing on magnetic anisotropy and linear deformation is considerable.
\end{abstract}

Recently, a great deal of attention has been paid to the magnetic anisotropy induced by stress annealing. ${ }^{1-3}$ It has been proposed that the stress-induced anisotropy $K$ is composed of two contributions, $K=K_{\text {an }}+K_{\text {plast }}$, where $K_{\text {an }}$ originates from the anelastic and $K_{\text {plast }}$ from the plastic deformation of the ribbon, ${ }^{4}$ when annealed under stress. From measurements of induced magnetic anisotropy and viscoelastic properties, ${ }^{5-7}$ very similar characteristics are deduced for $K_{\mathrm{an}}$ and $K_{\text {plast }}$ and the respective anelastic and plastic deformations $\epsilon_{\text {an }}$ and $\epsilon_{\text {plast }} . K_{\text {an }}$ is recoverable in the same manner as $\epsilon_{\mathrm{an}}$ and it is "frozen in" when the sample is cooled down with the applied stress. On the other hand, $K_{\text {plast }}$ is not recoverable and decreases with preannealing as $\epsilon_{\text {plast }}$ does. Other characteristics of $\epsilon_{\mathrm{an}}$ and $\epsilon_{\text {plast }}$ can be found in Refs. 8 and 9.

The usual procedure for annealing a sample is to treat it in a controlled atmosphere furnace in order to avoid oxidation during the annealing time (about $1 \mathrm{~h}$ ). Very recently, annealing by means of a short current pulse flowing through the sample has been shown to produce results in good agreement with the usual procedure. ${ }^{10-13}$ This "flash annealing" allows very short heating and cooling times ranging from some milliseconds to a few seconds. Because the time is so short "flash annealing" can be performed in air without an appreciable oxidation of the sample.

In this work we report the first simultaneous observations of the magnetic anisotropy and the viscoelastic deformation obtained by applying a tensile stress during the "flash annealing" of amorphous ribbons of composition $\left[\mathrm{Co}_{1-x}(\mathrm{FeNi})_{x}\right]_{75} \mathrm{Si}_{15} \mathrm{~B}_{10} \quad(0.1<x<0.7)$. The samples were ribbons $8 \mathrm{~cm}$ long and about $10^{-4} \mathrm{~cm}^{2}$ in cross section. Characteristic parameters of the samples have been pub- 
lished elsewhere. ${ }^{13}$ The experimental setup has been described in Ref. 12. The equipment allows a tensile stress to be applied while an electrical current flows through the sample and magnetic measurements can be performed in situ. The device has been improved by means of an air-cored linear variable differential transformer (LVDT) which allows one to determine the elongation of the sample $(\Delta l)$. The sensivity of the LVDT is about $1-\mu \mathrm{m}$ elongation and $1.5 \times 10^{-5}$ deformation $(\epsilon=\Delta l / l)$ of the sample.

The temperature of the sample during the flash annealing was determined by comparing the saturation magnetization under the current pulse with the value obtained by heating the sample in a conventional furnace. ${ }^{7}$ Temperature stabilization occurs in about $2 \mathrm{~s} ; 5$ - and 10 -s pulses were used in all the treatments and the temperature after stabilization was considered as the temperature of the sample during the annealing. Temperatures higher than the Curie temperature were determined by linear extrapolation of the temperaturecurrent curve. About $50^{\circ} \mathrm{C}$ extrapolation was needed in most cases.

Two kinds of experiments were performed, using asquenched and preannealed ribbons, respectively. The samples were preannealed for $10 \mathrm{~s}$ at the temperatures indicated in Fig. 1. In both cases, 5-s current pulses of increasing inten-
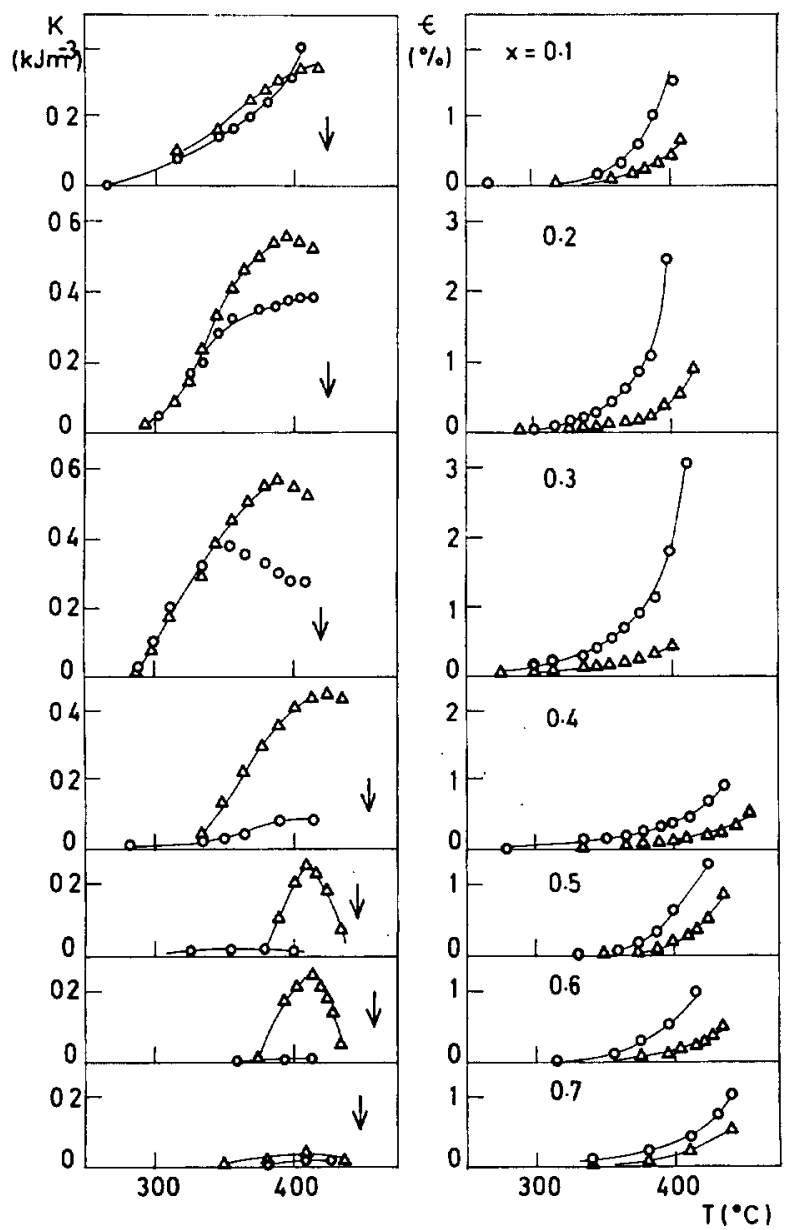

FIG. 1. Induced magnetic anisotropy and plastic deformation in samples of composition $\left[\mathrm{Co}_{1-x}(\mathrm{FeNi})_{x}\right]_{75} \mathrm{Si}_{15} \mathrm{~B}_{10}$ produced by means of 5 -s current pulses under 600-MPa stress. (O): As-quenched ribbons: $(\Delta)$ : samples preannealed for $10 \mathrm{~s}$ at the temperature indicated by the arrow. Current densities between $3 \times 10^{7}$ and $5 \times 10^{7} \mathrm{~A} \mathrm{~m}^{-2}$ have been used. sity were applied to the stressed ribbons. Current densities between $3 \times 10^{7}$ and $5 \times 10^{7} \mathrm{~A} \mathrm{~m}^{-2}$ flowing through samples of about $1 \Omega$ in $1 \mathrm{~cm}$ length were used in the treatments. The induced anisotropy and the linear deformation were determined after unloading the samples at room temperature. The measurements revealed a hard ribbon axis anisotropy which was determined from the saturation field $H^{k}$ (Ref. 5) as

$$
K=\frac{1}{2} \mu_{0} M_{s} H^{k} .
$$

After this treatment the samples were allowed to relax by applying to the unloaded sample a current pulse of the highest intensity. The remaining anisotropy, $K_{\text {plast }}$, could not be determined with our equipment, because the ribbon axis was the easy axis of magnetization, but its existence was deduced from the squareness of the hysteresis loop. ${ }^{2}$

Before relaxation we found that the induced anisotropy passes through a maximum as a function of annealing temperature in as-quenched and preannealed samples, with the exception of $x=0.1$ which the induced anisotropy continuously increases with annealing temperature. This maximum has been interpreted ${ }^{4}$ as arising from the competition between the anelastic $K_{\text {an }}$ and the plastic $K_{\text {plast }}$ components of the induced anisotropy. The anelastic component is a hard ribbon axis anisotropy and dominates at low temperature while the plastic component, which has an easy axis parallel to the ribbon, appears at higher annealing temperature. For $x=0.1$, the plastic anisotropy was found to give a hard ribbon axis, so that the maximum does not appear. For $x<0.4$, and except for the sample $x=0.1$ at high annealing temperatures, the preannealed ribbons show a higher induced anisotropy than the as-quenched ones. At low temperature, however, the value of $K$ is almost the same in both as-quenched and preannealed samples. In as-quenched ribbons no magnetic anisotropy is induced for $x>0.4$, but in preannealed ribbons a magnetic anisotropy is induced in a wider composition range $(0.1<x<0.7)$. For $x>0.7$, magnetic anisotropy can be induced neither perpendicular nor parallel to the ribbon axis. This is true both in as-quenched and in preannealed ribbons. The isochronal variation of the induced anisotropy, for $x=0.3$, studied by flash annealing agrees with that obtained in a conventional procedure. ${ }^{13}$ There are no previous data for the other compositions studied in this work.

In as-quenched as well as in preannealed ribbons, the induced anisotropy and a considerable linear deformation appear together. Figure 2 shows that the composition dependence of the induced magnetic anisotropy and the plastic deformation is similar, revealing a higher deformation for those compositions with a higher induced anisotropy. The composition dependence of the anisotropy induced by flash annealing of preannealed samples is the same as previously obtained in a conventional furnace. ${ }^{13}$ No previous data exist on as-quenched samples of these compositions.

The effect of the preannealing on the induced anisotropy is considerable especially in the range $x>0.4$. In order to study this influence in greater detail, different preannealing temperatures were used (Fig. 3). The composition $x=0.5$ and 0.2 have been chosen for this study because for $x=0.5$ 

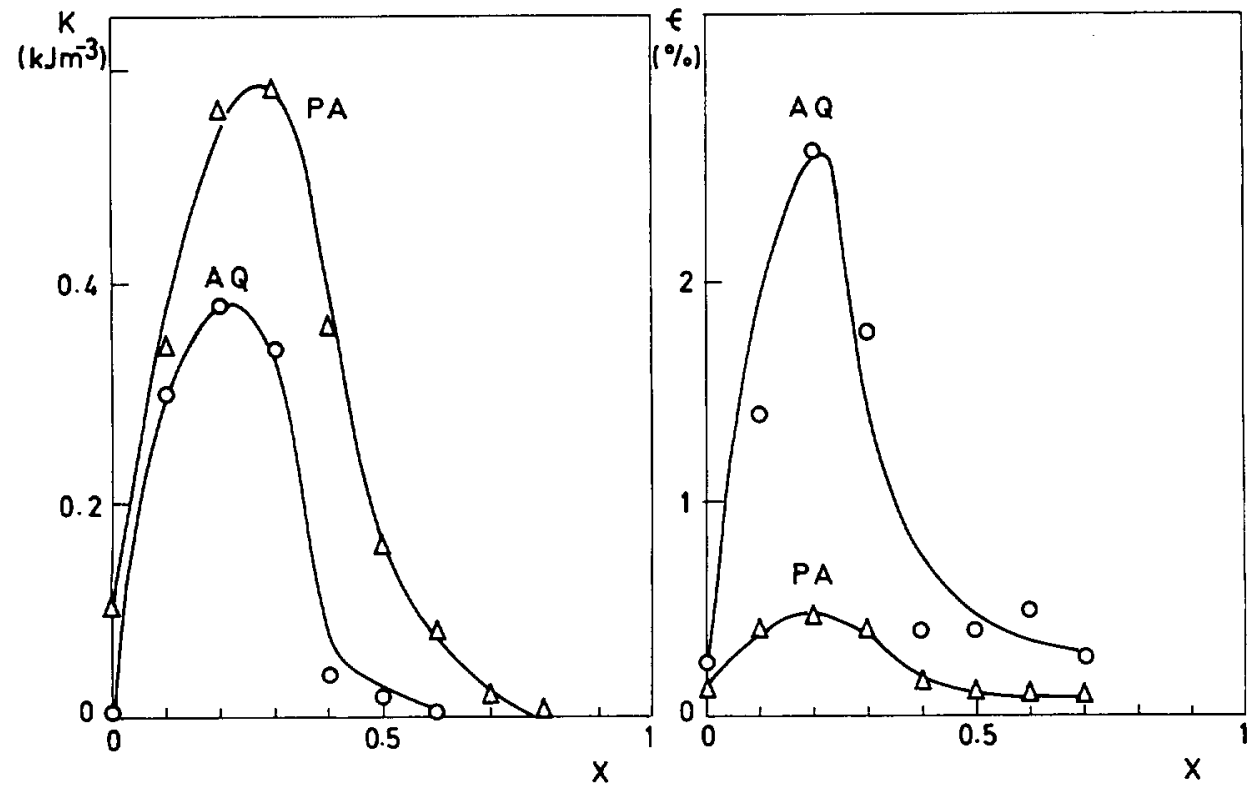

FIG. 2. Composition dependence of the induced magnetic anisotropy and the plastic deformation after $600-\mathrm{MPa}$ stress annealing at $395^{\circ} \mathrm{C}$ in $5 \mathrm{~s} .(\mathrm{O})$ : As-quenched ribbons; $(\Delta)$ : after preannealing for $10 \mathrm{~s}$ at $420^{\circ} \mathrm{C}$. Current densities used in the stress annealing and preannealing were about $4 \times 10^{7}$ and $4.5 \times 10^{7} \mathrm{~A} \mathrm{~m}^{-2}$, respectively. the magnetic anisotropy is only induced if the sample has been preannealed, whereas for $x=0.2$ the magnetic anisotropy is induced in both as-quenched and preannealed samples.

The induced anisotropy passes through a maximum as a function of the stress annealing temperature in all the cases. The magnitude of the maximum is higher for samples preannealed at higher temperatures or during longer times, and the deformation decreases in both cases. A decrease of the magnitude of the plastic anisotropy is believed to occur in
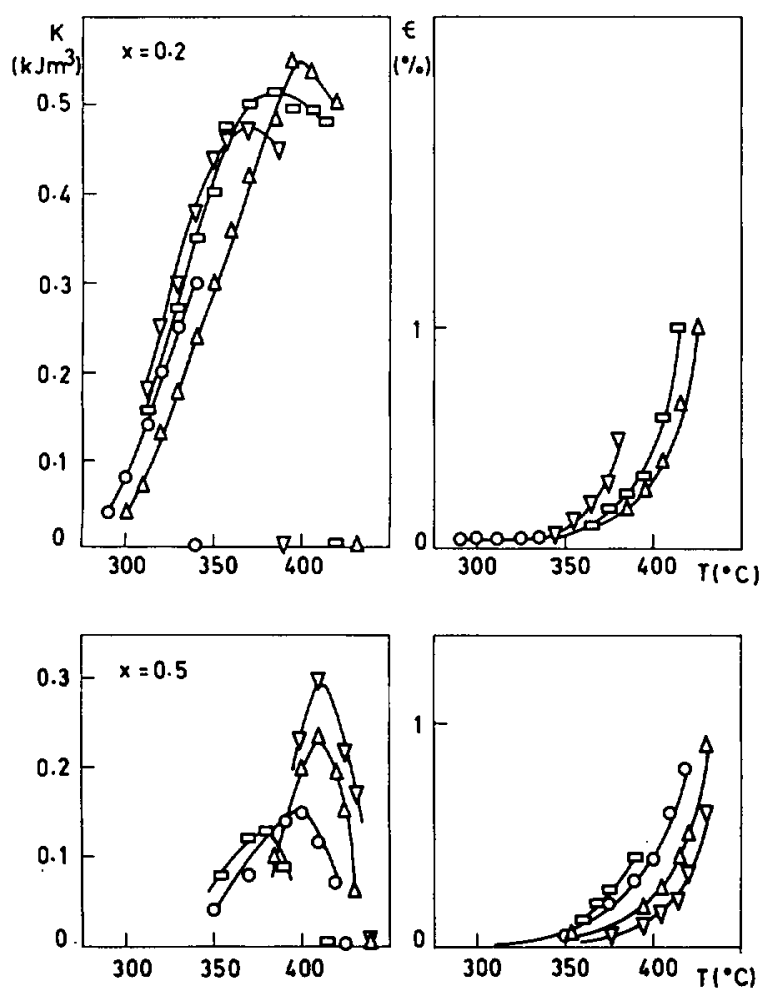

FIG. 3. Dependence of the induced magnetic anisotropy and the plastic deformation on the preannealing temperature. 10-s of preannealing has been used. The $x=0.5$ sample was furthermore exposed to 30 -s annealing $(\nabla)$. Preannealing temperatures are indicated on the temperature axis. these samples thereby giving less reduction of the net induced anisotropy.

The maximum of the induced anisotropy is obtained at higher temperatures for the samples preannealed at higher temperatures. This behavior can be interpreted as arising from a decrease of the atomic mobility as the samples are preannealed at higher temperature, a fact well established from the increase of viscosity upon annealing. ${ }^{14}$ On the other hand, a further increase of the preannealing time does not change the temperature of the maximum in the sample with $x=0.5$ although the deformation, and thus the atomic mobility, still decreases. Finally, it can be said that, for $x<0.4$ samples $(x=0.2)$, the effect of the preannealing is just to reduce the plastic anisotropy contribution, but in $0.4<x<0.7$ samples $(x=0.5)$ it is very surprising to find that the preannealing has two effects. First, preannealing is necessary, for the anelastic and plastic components to be induced by stress annealing. Second, increasing the preannealing temperature or time reduces the plastic component, resulting in a higher net hard ribbon axis induced anisotropy.

In conclusion, it has been shown that flash annealing under stress is an easy way to induce magnetic anisotropy. This procedure is faster and simpler than conventional treatments and the induced deformation can also be determined in situ with a suitable device. There is a close relation between the induiced magnetic anisotropy and the deformation. A higher deformation occurs in samples in which the induced anisotropy is higher and the effect of preannealing is considerable on both induced anisotropy and deformation.

${ }^{1}$ O. V. Nielsen and H. J. V. Nielsen, Solid State Commun. 35, 281 (1980), ${ }^{2}$ O. V. Nielsen and H. J. V. Nielsen, J. Magn. Mater. 22, 21 (1980).

${ }^{3}$ O. V. Nielsen, A. Hernando, V. Madurga, and J. M. Gonzalez, J. Magn. Magn. Mater. 46, 341 (1985).

${ }^{4}$ O. V. Nielsen, J. Magn. Magn. Mater. 36, 81 (1983).

${ }^{5}$ O. V. Nielsen, L. K. Hansen, A. Hernando, and V. Madurga, J. Magn. Magn. Mater. 36, 73 (1983).

${ }^{6} \mathrm{O}$. V. Nielsen, H. J. V. Nielsen, T. Masumoto, and H. M. Kimura, J. Magn. Magn. Mater. 24, 88 (1981). 
${ }^{7}$ V. Madurga, A. Hernando, and O. V. Nielsen, J. Phys. E. 17, 813 (1984).

${ }^{8}$ A. I. Taub and F. E. Luborsky, Acta Metall. 29, 1939 (1981).

${ }^{9}$ F. Spaepen and A. I. Taub, in Amorphous Metallic Alloys (Butterworths, London, 1983), Chap. 13.

${ }^{10}$ T. Jagielinski, IEEE Trans. Magn. MAG-19, 1925 (1983).

${ }^{11}$ M. R. T. Gibbs, D. H. Lee and J. E. Evetts, IEEE Trans. Magn. Mag-20,
1373 (1984)

${ }^{12}$ J. M. Barandiarán, A. Hernando, and O. V. Nielsen, IEEE Trans. Magn. MAG-22, 1864 (1986).

${ }^{13}$ O. V. Nielsen, J. M. Barandiarán, A. Hernando, and V. Madurga, J. Magn. Magn. Mater. 49, 124 (1985).

${ }^{14}$ A. I. Taub and F. Spaepen, Scr. Metall. 13, 195 (1979).

\title{
The thin-film reaction between $\mathrm{Ti}$ and thermally grown $\mathrm{SiO}_{2}$
}

\author{
J. C. Barbour, A. E. M. J. Fischer, and J. F. van der Veen \\ FOM Institute for Atomic and Molecular Physics, Kruislaan 407, 1098 SJ Amsterdam, The Netherlands
}

(Received 5 March 1987; accepted for publication 27 May 1987)

\begin{abstract}
Medium-energy ion scattering was used to examine the reaction between a thin Ti film $\left(4 \times 10^{15} \mathrm{Ti}\right.$ atoms $\left./ \mathrm{cm}^{2}\right)$ and a thermally grown $\mathrm{SiO}_{2}$ substrate. The reaction was monitored after deposition at room temperature and after annealing, up to a temperature of $800^{\circ} \mathrm{C}$. During deposition, oxygen from the $\mathrm{SiO}_{2}$ substrate dissolves into the Ti film (up to $38 \%$ oxygen) without the occurrence of Ti-Si intermixing. Heating of the sample causes a loss of oxygen but no loss of titanium. Instead, islands of titanium silicide and areas of bare Si surface are formed after an $800^{\circ} \mathrm{C}$ anneal for $250 \mathrm{~min}$. A comparison of the room-temperature reaction for the ternary Ti-O-Si system and the binary Ti-Si system is also given.
\end{abstract}

The titanium-silicon system is important in very-largescale integration (VLSI) because $\mathrm{TiSi}_{2}$ is stable with respect to phase transformations at high temperatures and $\mathrm{TiSi}_{2}$ has a low resistivity (about $20 \mu \Omega \mathrm{cm}$ ). ${ }^{1}$ For these reasons, titanium silicide is a good candidate as interconnect or gate material in contact with $\mathrm{a} \mathrm{SiO}_{2}$ insulator in thin-film device fabrication. Yet, little work with high depth resolution has been done to study the thermal reaction of a thin Ti film (less than 10-nm film thickness) with thermally grown $\mathrm{SiO}_{2}$. In this communication we present the first use of medium-energy ion scattering (MEIS), with 0.4-nm-depth resolution, to study the reaction between a thin film of Ti and a thin film of $\mathrm{SiO}_{2}$.

Kuiper et al. ${ }^{2}$ have investigated thick layers of $\mathrm{TiSi}_{2}$ and found that oxidation of $\mathrm{TiSi}_{2}$ at $500-600^{\circ} \mathrm{C}$ in $10^{-6}-10^{-4}$ Torr $\mathrm{O}_{2}$ causes a loss of titanium, which is thought to be in the form of a volatile titanium oxide. However, direct evidence for the volatilization of $\mathrm{TiO}_{x}$ from bulk $\mathrm{TiO}$ or $\mathrm{TiO}_{2}$ has not been reported. Instead, Brillson et al. ${ }^{3}$ report a loss of oxygen (also thought to be a volatile $\mathrm{TiO}_{x}$ ) in the solid-state reaction between $\mathrm{Ti}$ and $\mathrm{SiO}_{2}$. The reaction products in the Ti-SiO ${ }_{2}$ couple are $\mathrm{Ti}_{5} \mathrm{Si}_{3}$ and TiO. If the model of Kuiper et al. ${ }^{2}$ is correct and the film thicknesses are small enough to allow escape of the volatile species, then pure Si or titanium silicide remains after volatilization of $\mathrm{TiO}_{x}$ from a thin-film $\mathrm{Ti}-\mathrm{SiO}_{2}$ reaction couple. As the size of devices gets smaller, this reaction could either be a problem when the degradation area and device area become similar in size or a novel method in creating oxide-free gate regions by selective volatilization of $\mathrm{TiO}_{x}$. Therefore, the presence of both $\mathrm{Ti}$ and oxygen were monitored with MEIS in order to test for the volatilization of $\mathrm{TiO}_{x}$ after thermal reaction of a $\mathrm{Ti}-\mathrm{SiO}_{2}$ couple.

First, a $\mathrm{SiO}_{2}$ layer (approximately $4 \mathrm{~nm}$ thick) was grown on a Si(111) wafer at $900^{\circ} \mathrm{C}$ in a flowing dry oxygen ambient. The oxidized substrate was then placed in a vacu- um chamber, with a base pressure of $5 \times 10^{-11}$ Torr, for in situ Ti deposition, annealing, and analysis. The sample was analyzed using $175-\mathrm{keV} \mathrm{He}^{+}$ion scattering in the shadowing and blocking geometry of the $(0 \overline{1} 1)$ scattering plane in order to reduce the scattering yield from the Si substrate.

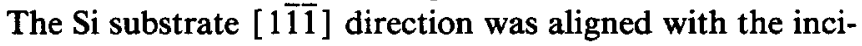
dent ion beam and the electrostatic analyzer (with an energy resolution $\delta E / E=4 \times 10^{-3}$ ) was aligned with the substrate [100] axis. The resultant scattering angle is $54.74^{\circ}$. (Medium-energy ion scattering, and the shadowing and blocking technique have been described in detail by van der Veen. ${ }^{4}$ ) In order to convert the MEIS integrated ion-scattering yield to an absolute number of atoms, with an accuracy of $5 \%$, the detection efficiency was measured by calibrating a thin film of $\mathrm{Ge}$ on Si with $2-\mathrm{MeV} \mathrm{He}^{+}$ion scattering. Neutralization of the ion beam was determined after each thermal treatment of the sample and low ion doses were used such that the ionbeam damage was negligible.

A Ti film $\left(4 \times 10^{15}\right.$ atoms $/ \mathrm{cm}^{2}$ which corresponds to a thickness of $0.7 \mathrm{~nm}$ ) was deposited onto the $\mathrm{SiO}_{2}$ in a background pressure of $5 \times 10^{-10}$ Torr. This amount of Ti was chosen to correspond to the point where a Ti overlayer gives complete coverage of a Si(111) surface. ${ }^{5}$ An example of the low substrate yield is shown in Fig. 1, in which the large signal-to-background ratio for both the oxygen peak and the Ti peak allows an accurate determination of the number of $\mathrm{Ti}$ and oxygen atoms. This figure shows that both $\mathrm{Si}$ and oxygen are at the surface before the titanium deposition (the surface position for each species is indicated by arrows). After deposition, the $\mathrm{Ti}$ and the oxygen peaks extend up to the surface position in the ion-scattering energy spectrum, but the Si peak is entirely shifted to a lower energy. Complete coverage of the $\mathrm{SiO}_{2}$ layer by $4 \times 10^{15} \mathrm{Ti}$ atoms $/ \mathrm{cm}^{2}$ is thus confirmed because the Si peak is shifted below the surface energy position, and the amount of this shift corre- 